\title{
Improved Ice Particle Optical Property Simulations in the Ultraviolet \\ to Far-infrared Regime
}

\author{
Lei Bi, Ping Yang
}




\section{Abstract}

To derive the bulk radiative properties of ice clouds, aircraft contrails and snow grains, which are fundamental to atmospheric radiative transfer calculations in downstream applications, it is necessary to accurately simulate the scattering of light by individual ice particles. An ice particle optical property database reported in 2013 (hereafter, TAMUice2013) is updated (hereafter, TAMUice2016) to incorporate recent advances in computation of the optical properties of nonspherical particles. Specifically, we employ the invariant imbedding Tmatrix (II-TM) method to compute the optical properties of particles with small to moderate size parameters. Both versions use the Improved Geometric Optics Method (IGOM) to compute the optical properties of large ice crystals, but TAMUice2016 improves the treatment of inhomogeneous waves inside the scattering particles in the case where ice is absorptive such as at infrared wavelengths. To bridge the gap between the extinction efficiencies computed from the II-TM and the IGOM, TAMUice2016 includes spectrally dependent higher order terms of the edge effect in addition to the first order counterpart considered in TAMUice2013. Furthermore, the differences between TAMUice2013 and TAMUice2016 are quantified with respect to the computation of the bulk optical properties of ice clouds.

Keywords: Ice particles, optical properties, database, ultraviolet through far-infrared 


\section{Introduction}

Ice particles and snow grains in the terrestrial atmosphere and cryosphere are critical components of the Earth's climate system. Atmospheric ice particles exist in the forms of cirrus clouds, aircraft contrails, type II polar stratospheric clouds (PSCs), and snowflakes. Ice particles can also coexist with supercooled water droplets in mixed-phase clouds. The optical properties of ice particles are fundamental to atmospheric radiative transfer simulations and remote sensing implementations [1-4]. For this reason, substantial computational efforts have been made to compute the optical properties of various ice crystals [5-12] and to develop data libraries in different spectral regimes with consideration of various particle microphysical properties, including particle size, habit and surface texture [13-17]. Furthermore, the bulk optical properties of an ensemble of ice crystals in ice clouds have been developed for practical applications [e.g., 18-20].

An ice optical property database (hereafter, TAMUice2013) has been developed [21] for 11 representative ice crystal habits, namely column, hollow column, plate, droxtal, 5-plate aggregate, 10-plate aggregate, 8-column aggregate, solid bullet rosette, hollow bullet rosette, sphere, and spheroid, over a spectral range of 0.2-100 $\mu \mathrm{m}$. TAMUice2013 was an effort based on the concepts of two previous datasets [14,22] with tremendous new computational effort to improve the computational consistency in terms of size-bin resolution and detailed particle morphologies. In addition, the degree of particle surface roughness is considered as an additional morphological parameter in TAMUice2013. To compute the optical properties of each single ice particle, in TAMUice2013 the discrete dipole approximation (DDA) 
method [23, references cited therein] was used for small size parameters up to approximately 20 (the specific value varies with the incident wavelength) and the Improved Geometric Optics Method (IGOM, [10]) for size parameters exceeding 20. The IGOM results are semiempirically adjusted to take into account the edge effect, a term introduced within the framework of a semi-classical scattering theory [24]. TAMUice2013 has already been extensively used in remote sensing applications and radiative forcing studies involving ice clouds (e.g. [25, 26]). Furthermore, the 8-column aggregate model with severe surface roughness is used for the Moderate Resolution Imaging Spectroradiometer (MODIS) Collection 6 (C6) operational ice cloud property retrieval product [27]. A pronounced benefit of the MODIS C6 ice crystal habit model is that it gives rise to consistent optical thickness retrievals using either an algorithm based on visible and near-infrared bands or an algorithm based completely on thermal infrared bands [28].

With feedback from the research community and recent advances in light-scattering computational techniques, we update TAMUice2013. To improve the numerical accuracy of the updated database (hereafter, TAMUice2016), we use a new computational technique, the invariant imbedding T-matrix (II-TM) method, to improve the accuracy in computing the single-scattering properties of randomly oriented ice particles with small to moderate size parameters. In the Christiansen bands ([29, 30] and references therein), where the real part of the refractive index is close to unity and the imaginary part of the refractive index is relatively large, the IGOM accuracy for large particle sizes is significantly improved. In 
particular, we substantially improve the treatment of inhomogeneous waves within absorbing ice particles when the imaginary part of the ice refractive index is not zero.

This paper is organized as follows. In Section 2, we briefly summarize the II-TM for determining the single-scattering properties of ice particles. We focus on summarizing the intrinsic strengths of the II-TM, regarding enhancing the accuracy of the single-scattering simulations involving ice crystals. We also demonstrate an improved treatment of inhomogeneous waves in the case of absorbing particles for computing the extinction efficiency. The formalism of the new IGOM implementation is included in Appendix A. Furthermore, we also present a rigorous proof of the asymptotic extinction efficiency in the context of an electric-field volume integral equation in Appendix B. In Section 3, we compare the single-scattering properties of ice particles in TAMUice2013 and TAMUice2016. The summary and conclusions of this study are given in Section 4.

\section{Computational Improvements}

TAMUice2016 uses the basic TAMUice2013 methodology based on a synergistic combination of different computational methods. However, TAMUice2013 used the DDA method (specifically, the ADDA code developed in [23]) for small to moderate size parameters. TAMUice2016 uses the II-TM for that size parameter regime instead, and incorporates some new improvements of the IGOM in the infrared spectral region where ice crystals are significantly absorptive. 
The basic concept of the II-TM is described by Johnson [31], and further theoretical development and a numerically stable implementation of this method are reported by $\mathrm{Bi}$ and Yang [11] in the case of general nonspherical particles without axial symmetry. It is worth noting that averaging the optical properties with the T-matrix technique under the random orientation condition can be performed analytically, as proved by Mishchenko [32]. This computational advantage is not provided by numerical methods such as DDA or the finitedifference time domain (FDTD) method [9, 33, 34]. Furthermore, in DDA or FDTD, the number of particle orientations required to appropriately represent the random orientation condition increases significantly with the size parameter and is also strongly sensitive to the imaginary part of the refractive index and particle's shape [35]. In particular, for highly absorptive particles, the near-backscattering phase function should be flat for randomly oriented particles within the geometric optics framework; however, if the number of orientations is insufficient, the near-backscattering phase function exhibits artifact structures or incorrectly enhanced backscattering. For TAMUice2016, in the case of absorbing convex ice particles, the size parameter region where the contribution is negligible from higher order refracted waves is identified based on a simple formula reported by Bi and Yang [36], so the size parameter domain is divided into two subdomains for using the II-TM and IGOM, respectively. This improvements enhances the accuracy of the optical properties for small and moderate size parameters, and ensures a smooth transition from the II-TM regime to the IGOM large particle regime. 
The ray-tracing treatment of inhomogeneous waves in absorbing ice particles [37-39] is applied rigorously but in a simplified way as in the previous version of IGOM. The effect of an inhomogeneous wave is only taken into account in the determination of the directions of refracted rays in the scattering particle, although in an absorbing medium the electric field has a nonzero component along the ray propagation direction. The new numerical implementation of the IGOM is presented in Appendix A.

Furthermore, for an ice spheroid we employ a new formula developed by Bi and Yang [36] to compute the extinction efficiency. Although the formula is obtained semi-empirically by modifying the asymptotic formulation for a sphere based on the complex angular momentum theory, the formula for a spheroid is quite accurate in comparison with the results computed from the II-TM. For other ice crystal habits, TAMUice2016 continues to use the methodology in TAMUice2013, but includes higher order terms to bridge the extinction efficiencies computed from the II-TM and the IGOM.

\section{TAMUice2013/2016 Comparison}

In this section, we focus on analysis of the single-scattering properties reported in TAMUice2016 using the II-TM, and compare the results with the TAMUice2013 counterparts.

Fig. 1 shows a comparison between TAMUice2013 and TAMUice2016 for the asymmetry factor values of hexagonal ice columns. Specifically, the left panel shows the results 
computed from the ADDA+IGOM. The blue line depicts a dividing line in TAMUice2013, below and above which the ADDA and IGOM are employed, respectively. The middle panel shows improved computation based on a combination of the II-TM and IGOM in TAMUice2016. The blue line depicts a dividing line for the applications of the II-TM (below the dividing line) and IGOM (above the dividing line). The general patterns of TAMUice2013 and TAMUice2016 are similar, but some differences can be noticed. The right panel shows the differences defined in terms of (TAMUice2013-TAMUice2016)/ TAMUice $2016 \times 100 \%$. The relative differences between the two databases can be as large as $\pm 15 \%$, primarily in the resonance region when the radii of the circumscribed spheres of nonspherical ice crystals are comparable to the incident wavelength.

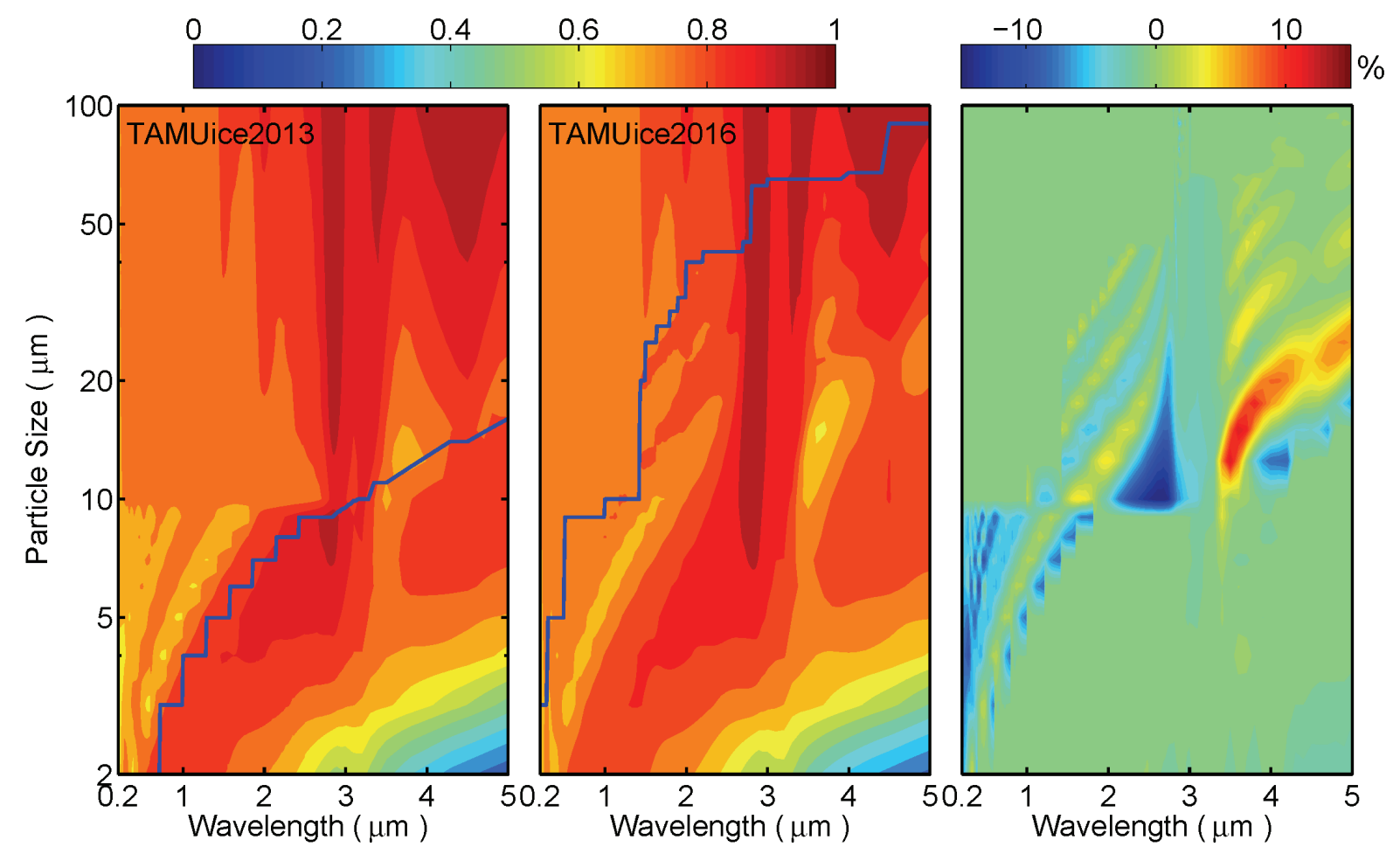

Fig. 1 Comparison of the asymmetry factor: (left panel) TAMUice2013 based on an ADDA+IGOM combination; (middle panel) TAMUice2016 based on an II-TM+IGOM 
combination; (right panel) relative differences defined in terms of (TAMUice2013TAMUice2016)/ TAMUice $2016 \times 100 \%$.

Fig. 2 shows the effect of including higher order terms. For absorptive ice particles, the contribution from higher-order rays to the scattering phase function decreases as the size parameter increases. If the higher-order contributions were negligible, the phase function computed from convex non-spherical particles would be much closer to those of spheres. Therefore, for randomly oriented convex particles, the asymptotic phase function in the direct backscattering direction can be written in the form

$$
P\left(180^{\circ}\right)=\frac{1}{Q_{\text {sca }}}\left|\frac{m-1}{m+1}\right|^{2},
$$

where $m$ is the complex refractive index and $\mathrm{Q}_{\text {sca }}$ is the scattering efficiency. To obtain the critical value of particle size below which the higher order contributions to the scattering phase function must be considered, we compare $\mathrm{P}\left(180^{\circ}\right)$ computed from the II-TM and Eq. (1), as shown in Fig. 2, for droxtal ice crystals [12]. Insignificant differences for large particle sizes (the specific values depend on the incident wavelength) indicate negligible contributions from high-order rays, because most energy associated with the first-order refracted rays is absorbed. 

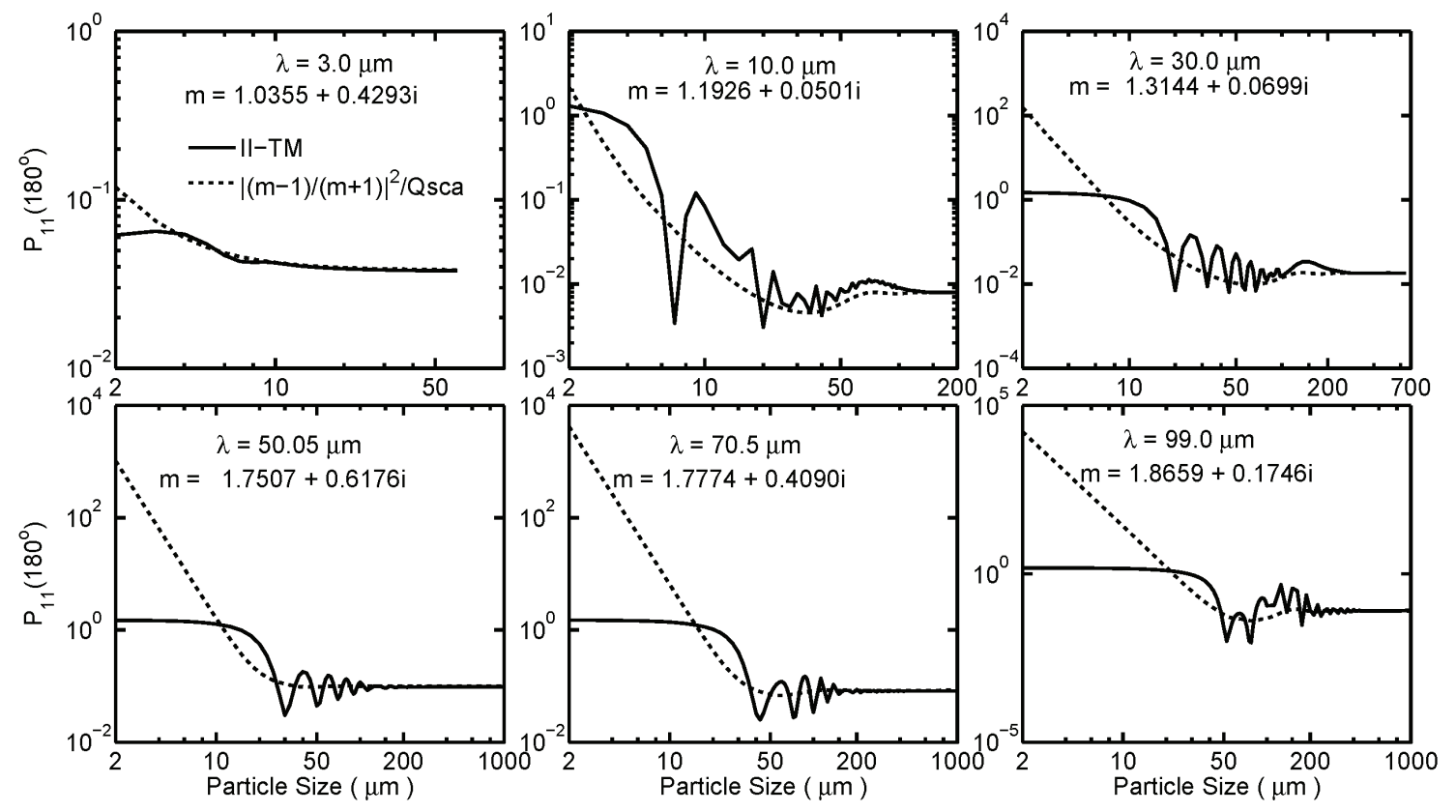

Figure 2. Backscattering phase function associated with droxtal ice crystals. The close agreement of the II-TM results and the dashed line for large (relative to the incident wavelength) particles indicates the region where higher-order ray contributions are negligible.

Fig. 3 illustrates the effect of the treatment of inhomogeneous waves on the extinction efficiency computed with the IGOM in the case of absorptive hexagonal ice columns, particularly in the spectral region where the real part of the refractive index is relatively small and the imaginary part of the refractive index is substantial. As shown in the left panel, the extinction efficiencies for a particle size of $10000 \mu \mathrm{m}$ from TAMUice2013 and TAMUice2016 show large differences at wavelengths near 2.91, 11.1, 44.5 and $48 \mu \mathrm{m}$. In the right panel, the extinction efficiencies at the five wavelengths as functions of particle size are depicted. To illustrate the asymptotic solutions by the geometric optics approach, the edge effect contribution to the extinction efficiency is not taken into account in Fig. 3. However, it 
must be clarified that the edge effect is incorporated into both TAMUice2013 and TAMUice2016.
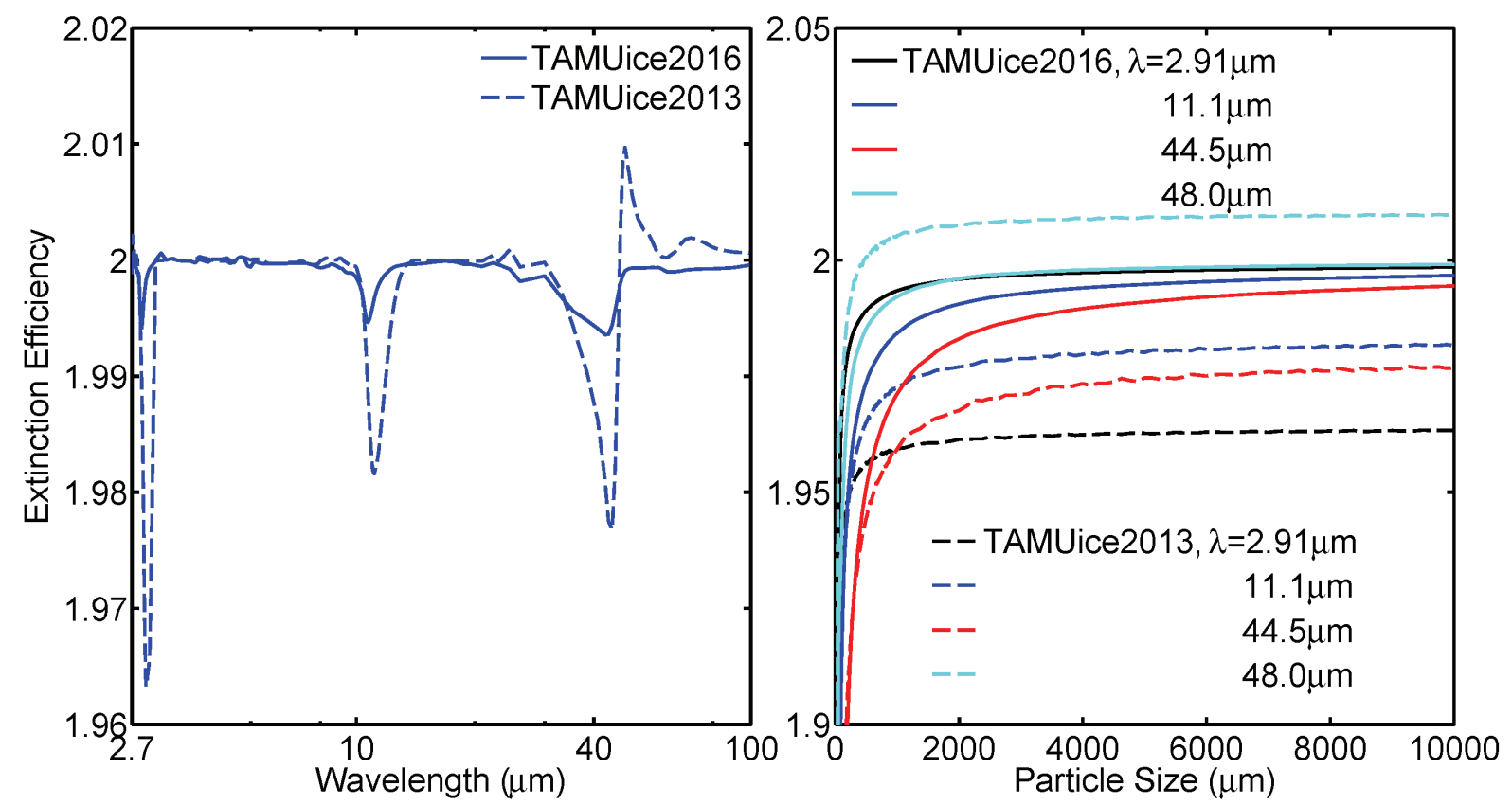

Figure 3. Left: spectral extinction efficiencies of randomly oriented hexagonal columns with a size of $10000 \mu \mathrm{m}$. Right: extinction efficiencies at four different wavelengths as functions of particle size.

Bi and Yang [36] developed an analytical formula to compute the extinction efficiency of spheroids with high accuracy, which is incorporated in TAMUice2016 computation. Fig. 4 shows the extinction efficiencies computed from the II-TM and the analytical formula across the entire particle size range for all wavelengths. The blue line depicts a dividing line for the applications of the II-TM (left of the dividing line) and IGOM (right of the dividing line). For large wavelengths, the II-TM is only applied for particle sizes smaller than $1000 \mu \mathrm{m}$. The 
general patterns in conjunction with different aspect ratios are similar, although absolute values are different with minor deviations in terms of local patterns. The II-TM essentially covers the entire resonance domain.
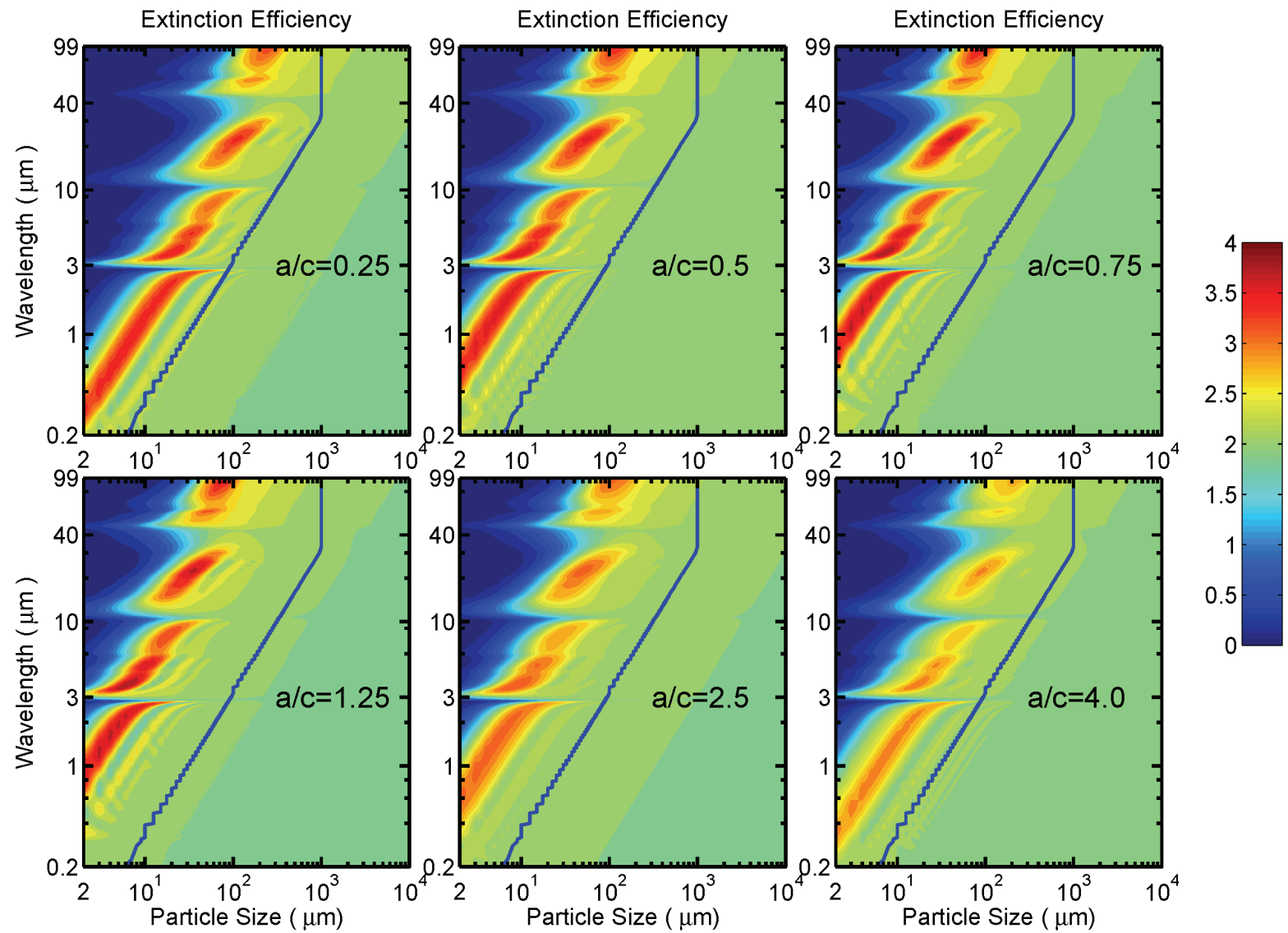

Figure 4. Contour plot of the extinction efficiencies of spheroids with different aspect ratio (a/c) values.

To illustrate clearly the transition of the extinction efficiencies computed with the II-TM to counterparts based on the analytical formula, Fig. 5 compares the extinction efficiencies for five selected wavelengths for six aspect ratio. The solid lines are computed with the II-TM, and the dashed lines are computed from the analytical formula. Note that there are no fitting 
coefficients in the formula, and that the transition from the II-TM solution to the results based on the analytic formula is smooth in all cases.
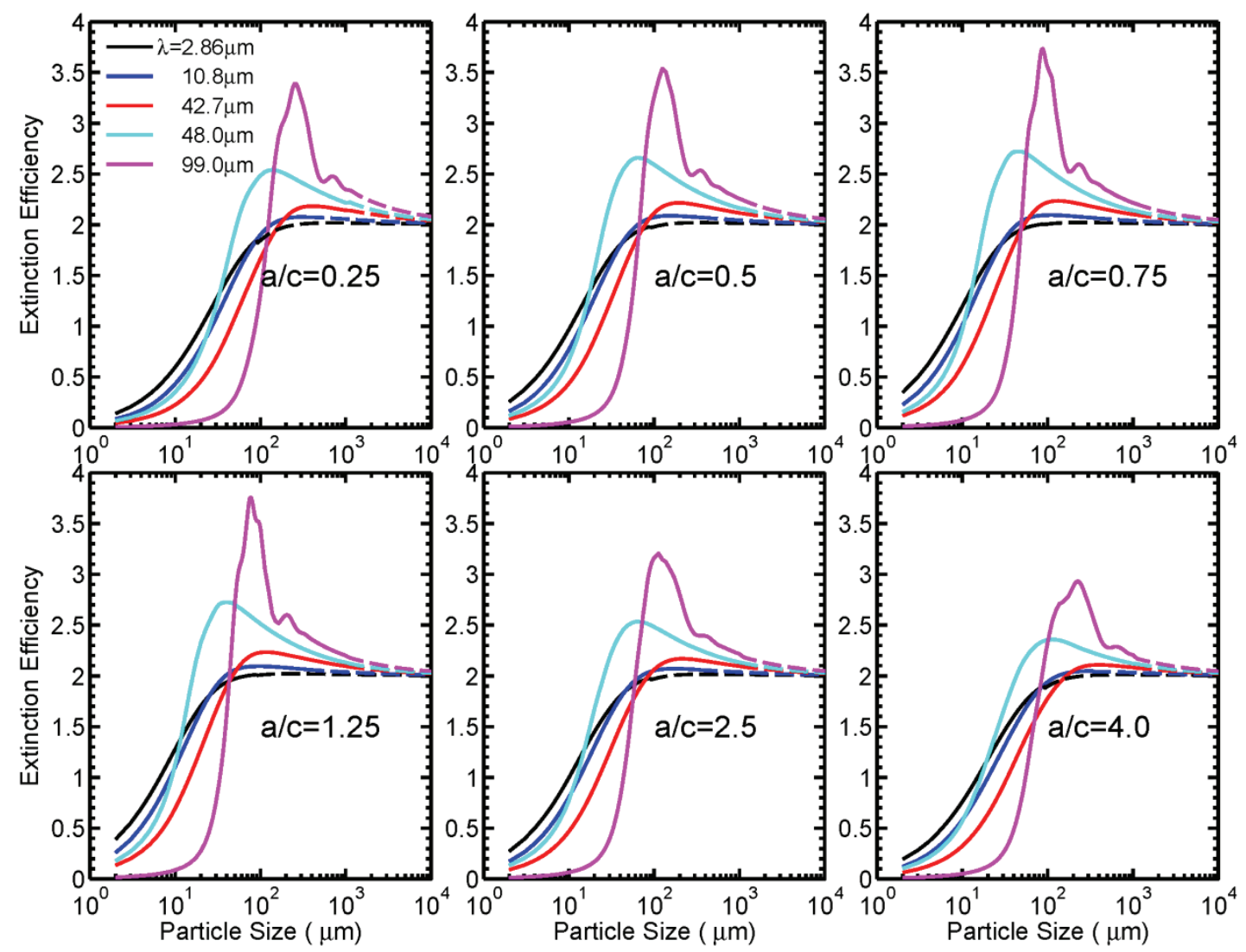

Fig. 5. Extinction efficiencies of spheroids with six aspect ratios. For each aspect ratio, five wavelengths are selected for comparison. The solid lines are computed from the II-TM, and the dashed lines are computed from an analytical formula.

To compare the edge effect of spheroids and spheres, Fig. 6 plots the ratio of the edge effect efficiency of a spheroid to that of a sphere across the entire wavelength spectrum for a particle size of $10000 \mu \mathrm{m}$. It is noted that the ratio changes significantly in some spectral regions where the real part of the ice refractive index is small and the corresponding 
imaginary part is large. For prolate spheroids $(\mathrm{a} / \mathrm{c}=0.25)$, the ratio is relatively large. For oblate spheroids $(\mathrm{a} / \mathrm{c}=1.25)$, the ratio is relatively small.

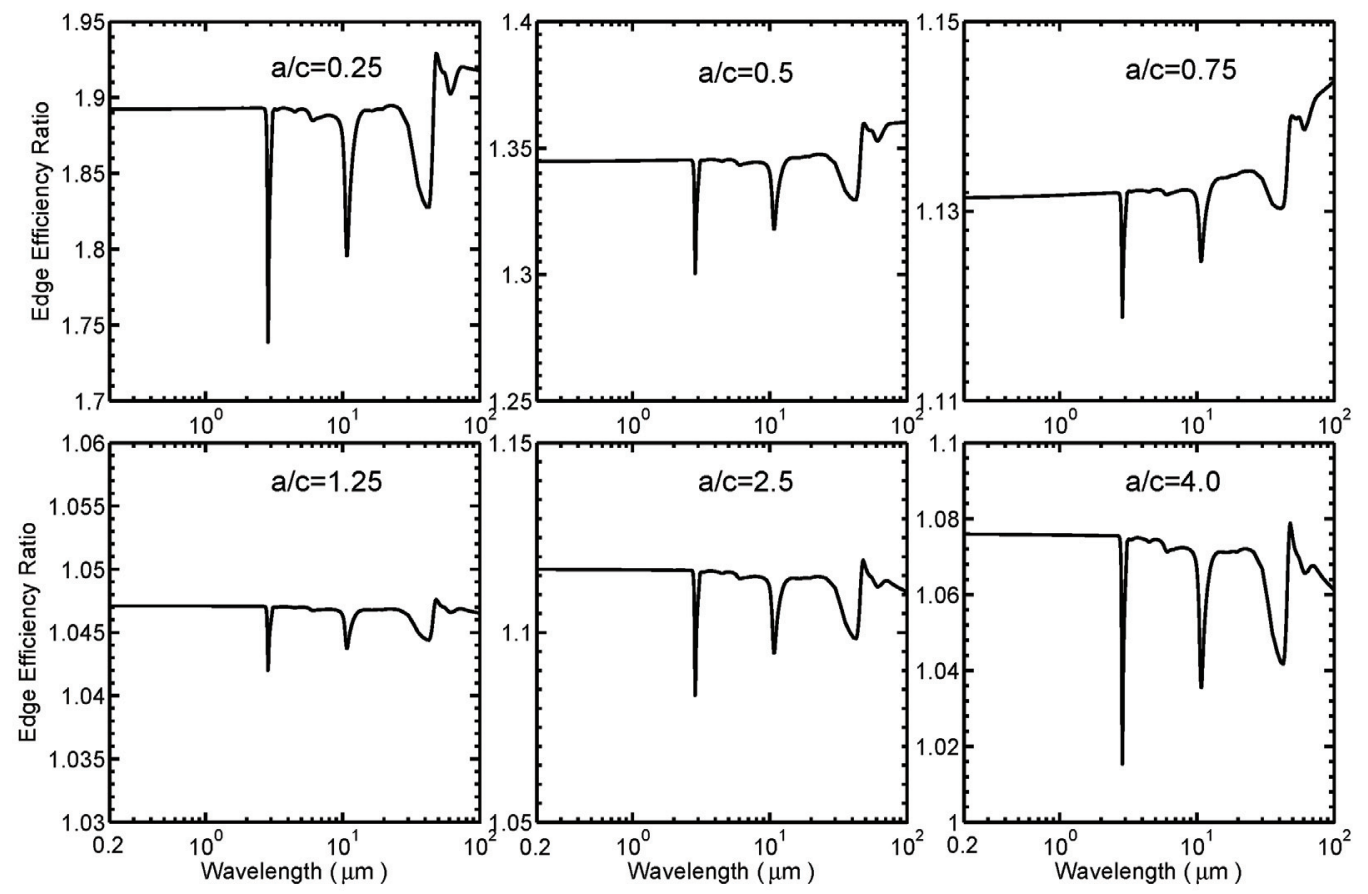

Fig.6. The ratio of spectral edge-effect efficiencies of spheroids over those of spheres.

To illustrate the impact of the differences between TAMUice2013 and TAMUice2016 on practical applications, we employ the MODIS C6 ice particle habit model (for particle morphology and size distribution ) [27] in conjunction with the two databases to compute the bulk optical properties. Figs. 7, 8, and 9 show the bulk extinction efficiency, single-scattering albedo, and asymmetry factor as functions of the effective particle size $\left(r_{e}\right)$, respectively, at two wavelengths $\lambda=0.65 \mu \mathrm{m}$ and $11 \mu \mathrm{m}$. From the left-upper panel in Fig. 7, it is noted that the bulk extinction efficiency based on TAMUice2016 approaches to 2 when the effective particle size is large (specifically, ${ }_{e}>80 \mu m$ ), whereas the TAMUice2013-based calculation 
shows larger values. According to the principle of geometric optics, the asymptotic extinction efficiency of a very large particle is 2 . Thus, TAMUice2016 is more accurate than TAMUice2013 from the perspective of the aforesaid physically rationale. However, the differences of the two results are small (specifically, smaller than 3\%), as evident from the upper-right panel of Fig. 7. In particular, the differences are less than 1\% when the effective radius is larger than $30 \mu \mathrm{m}$. The lower two panels of Fig. 7 show the comparison of the two results at an infrared wavelength of $11 \mu \mathrm{m}$. In this case, TAMUice2016-based results are slightly larger but the magnitudes of the differences are less than $1 \%$. For moderate and large particle sizes $\left(r_{e}>30 \mu m\right)$, the differences are essentially due to the improved treatment of the inhomogeneous waves inside ice particles whereas the improvements due to the use of the II-

TM in TAMUice2016 are response for the differences for small particle sizes $\left(r_{e}<30 \mu m\right)$.
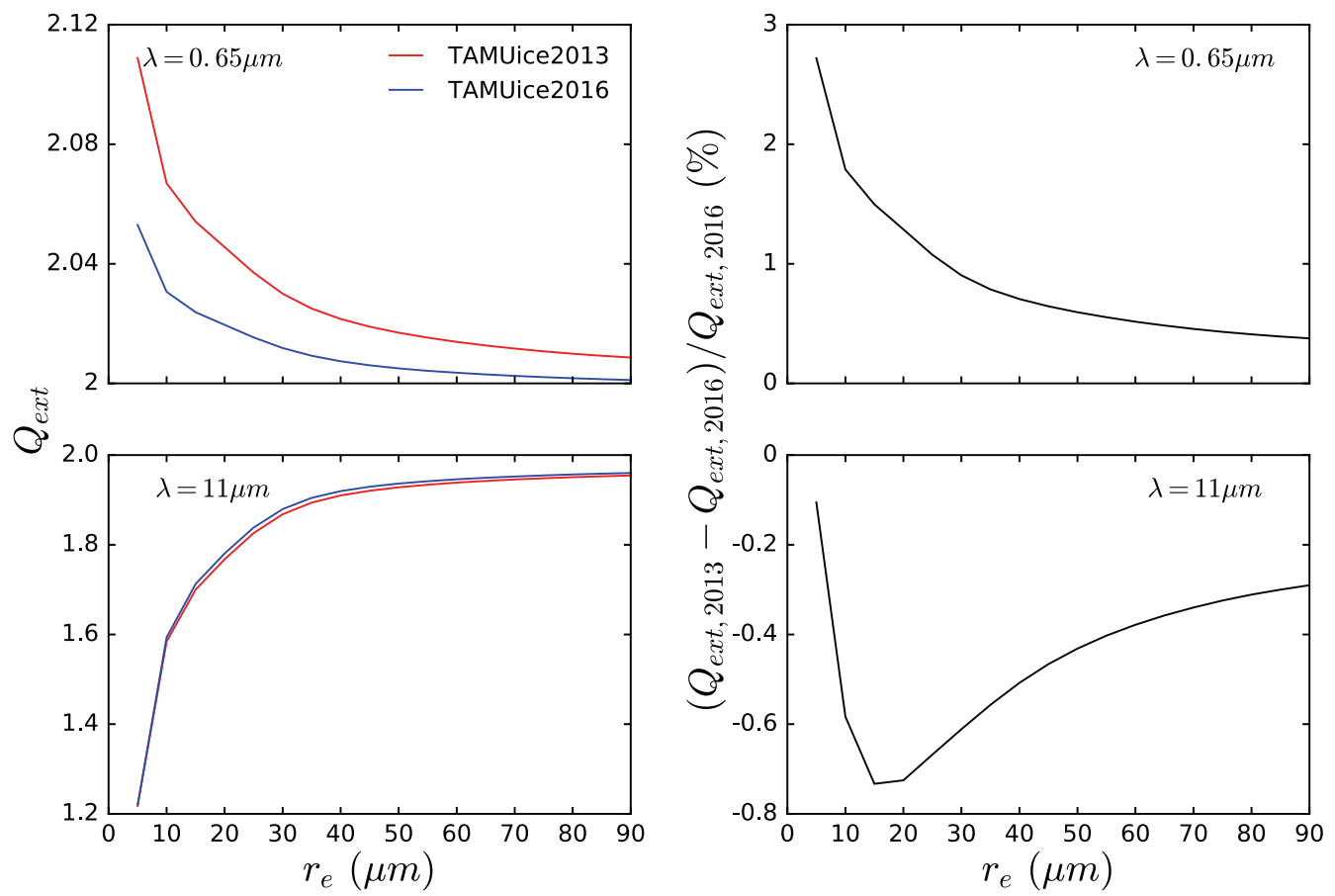
Fig. 7. The bulk extinction efficiencies computed from using the MODIS C6 ice habit model (for particle morphology and size distribution) [27] in conjunction with the TAMUice2013 and TAMUice2016 optical property datasets.

Fig. 8 shows the bulk single-scattering albedo. For wavelength $0.65 \mu \mathrm{m}$, both TAMice2013 and TAMU2016 based simulations give single-scattering albedo values that approach essentially to unity because ice crystals are not absorptive at this wavelength. At wavelength $11 \mu \mathrm{m}$, the TAMUice 2016 results slightly enhanced absorption (i.e., with slightly smaller single-scattering albedo), and the maximum of the differences is observed at $r_{e} \approx 20 \mu \mathrm{m}$.
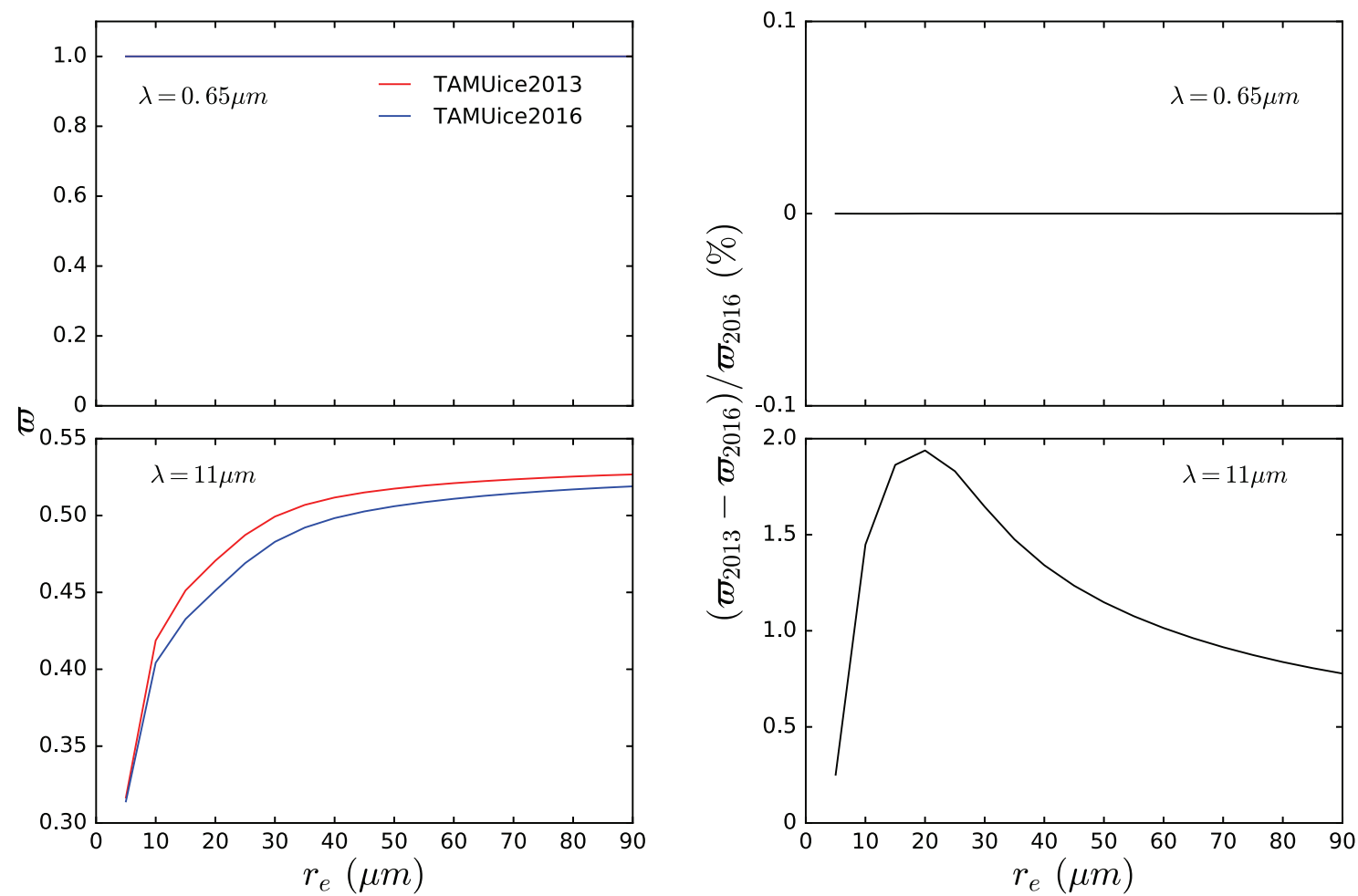

Fig. 8. Same as Fig.7, except for the bulk single-scattering albedo. 
Fig. 9 is similar to Figs. 7 and 8, but for the asymmetry factor. For the visible wavelength, the differences can be neglected, as evident from the upper panels of Fig. 8. For wavelength 11 $\mu \mathrm{m}$, the magnitudes of the differences are not larger than $1.2 \%$. However, in the infrared spectral regime, absorption dominates and the detailed phase function (thus, the asymmetry factor) is less important.
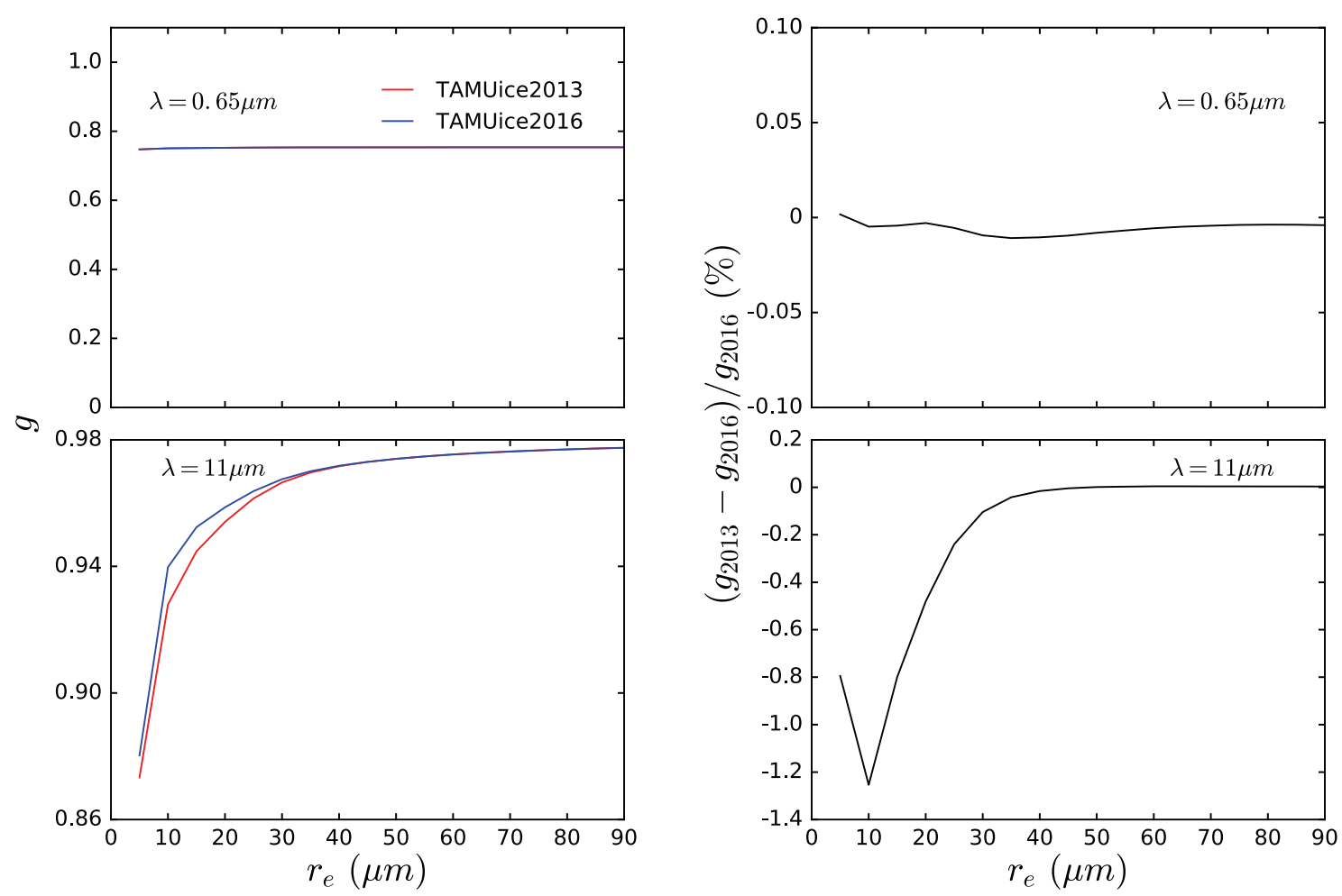

Fig. 9. Same as Figs. 7 and 8, except for the asymmetry factor.

From Figs. 7-9, it is expected that the impact of the differences between TAMUice2013 and TAMUice2016 is small for practical applications. For example, the MODIS C6 ice cloud property retrievals are based on a pair of a visible band and a near-infrared band [27]. The information content for retrieving ice cloud optical thickness is essentially from a visible band. It has been suggested that $\tau(1-\omega g)$ is invariant [27,40], which reduces to $\tau(1-g)$ at a 
visible wavelength for which the absorption of ice is negligible. According to this invariant quantity and Fig. 9, it is expected that the update on the ice crystal optical property database has little impact on the MODIS C6 ice cloud properties. Specifically, Fig. 10 shows the ratio $\tau_{\text {TAMUice2013 }} / \tau_{\text {TAMUice2016 }}$ (here $\tau_{\text {TAMUice2013 }}$ and $\tau_{\text {TAMUice2016 indicate the optical thickness values }}$ inferred from the radiometric observations made at a visible band centered at $0.65 \mu \mathrm{m}$ in conjunction with the use of the TAMUice2013 and TAMUice2016 datasets, respectively), which is given by $\left(1-\tilde{\boldsymbol{\omega}}_{\text {TAMUice } 2016} g_{\text {TAMUice2016 }}\right) /\left(1-\tilde{\boldsymbol{\omega}}_{\text {TAMUice } 2013} g_{\text {TAMUice2013 }}\right)$, as a function of the effective particle size. It can be estimated from Fig. 10 that $\left|\tau_{\text {TAMUice } 2013} / \tau_{\text {TAMUice } 2016}-1\right| \times 100 \%$ is less than $0.04 \%$ and the differences can be neglected for retrieving ice cloud optics thickness from observations in a visible band. 


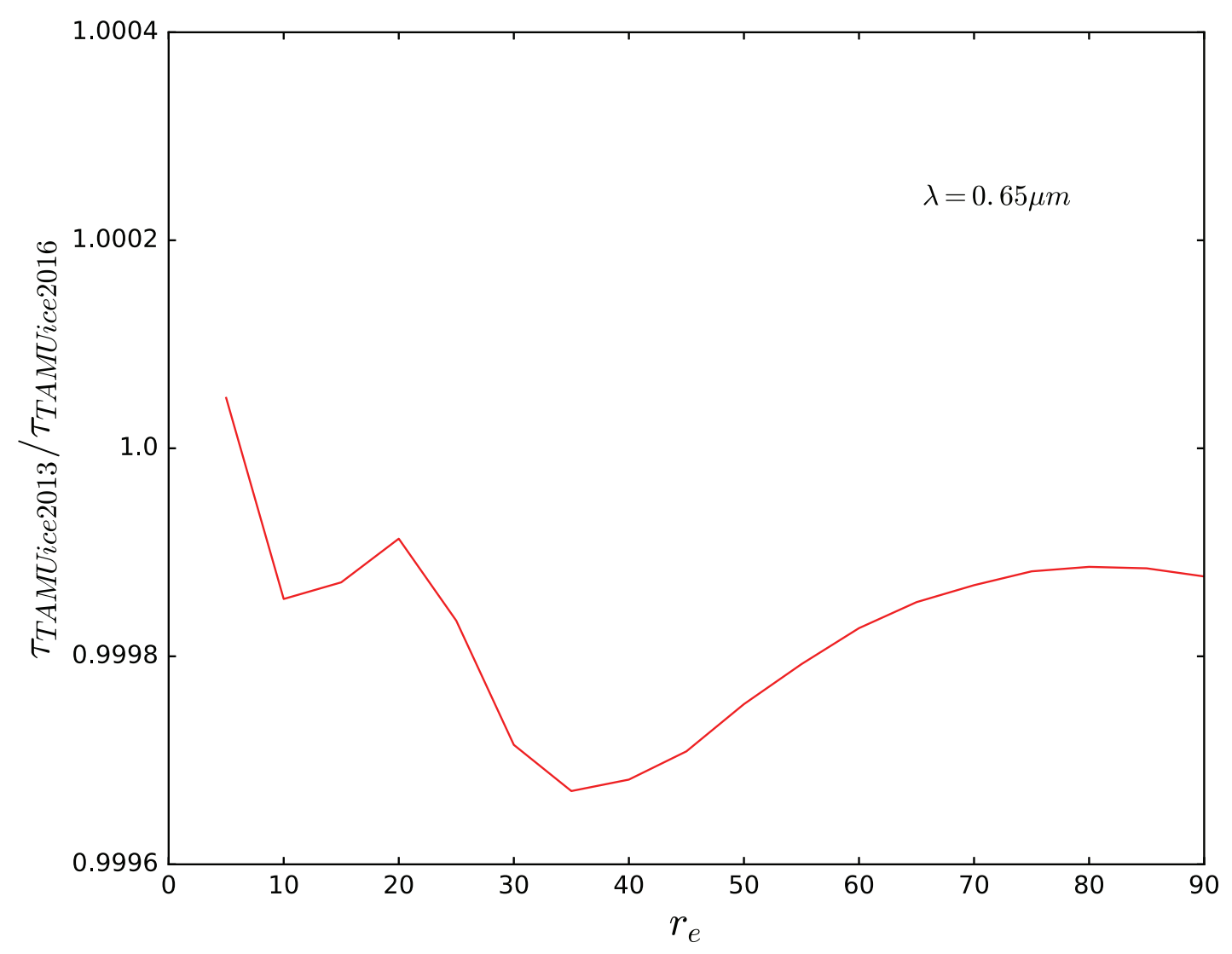

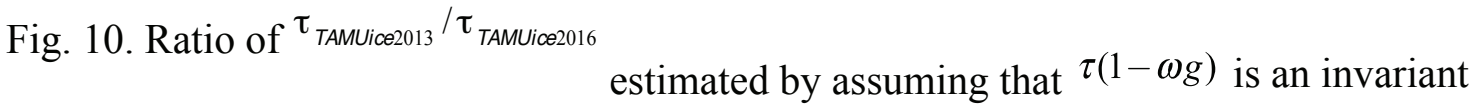

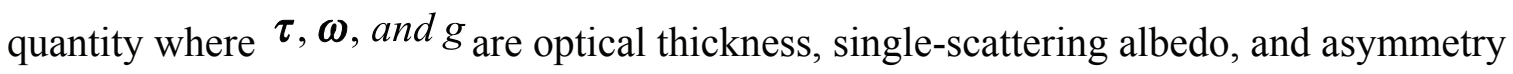

factor, respectively.

\section{Summary}

This effort updates a database of the optical database of the optical properties of ice crystals from a previous version referred to as TAMUice2013 to an updated version referred to as TAMUice2016. The new version improves the accuracy of the single-scattering properties of 
ice particles with small to moderate size parameters. In particular, the transition of the asymmetry factor from the electrodynamics wave domain to the geometric optics domain is much smoother. Furthermore, we have also extended the spectral region into the far-infrared and microwave region, which is reported in a separate paper [41].

\section{Acknowledgment}

This study was supported by the National Science Foundation (AGS-1338440) and partly by the endowment funds related to the David Bullock Harris Chair in Geosciences at the College of Geosciences, Texas A\&M University. Lei Bi's work presented in this paper was performed when he was at Texas A\&M University. The authors thank Mr. Jiachen Ding for help with Figs. 7-10. 


\section{References:}

1. Liou KN, Yang P. Light Scattering by Ice Crystals: Fundamentals and Applications. Cambridge, UK: Cambridge University Press; 2016.

2. Baran A J. A review of the light scattering properties of cirrus. J Quant Spectrosc Radiat Transf 2009;110:1239-60.

3. Baran A J. From the single-scattering properties of ice crystals to climate prediction: A way forward. Atmos Res 2012;112:45-69.

4. Yang P, Liou KN, Bi L, Liu C, Yi BQ, Baum BA. On the radiative properties of ice clouds: Light scattering, remote sensing, and radiation parameterization. Adv Atmos Sci 2015; 32(1):32-63, doi:10.1007/s00376-014-0011-z.

5. Macke A. Scattering of light by polyhedral ice crystals. Appl Opt 1993; 32:2780-88.

6. Muinonen K. Scattering of light by crystals: A modified Kirchhoff approximation. Appl Opt 1989; 28:3044-50.

7. Takano Y, Liou KN. Solar radiative transfer in cirrus clouds. Part I. Single scattering and optical properties of hexagonal ice crystals. J Atmos Sci 1989; 46:3-19.

8. Um JS, Mcfarquhar GM. Single-scattering properties of aggregates of bullet rosettes in cirrus. J Appl MeteorClimatol2007; 46:757-75

9. Yang P, Liou KN. Finite-difference time domain method for light scattering by small ice crystals in three-dimensional space. J Opt Soc Am A 1996; 13(10):2072-85.

10. Yang P, Liou KN. Geometric-optics-integral-equation method for light scattering by nonspherical ice crystals. Appl Opt 1996; 35:6568-84. 
11. Bi L, Yang P. Accurate simulation of the optical properties of atmospheric ice crystals with invariant imbedding T-matrix method. J Quant Spectrosc Radiat Transf 2014; $138: 17-35$

12. Zhang Z, Yang P, Kattawar GW, Tsay SC. Baum BA, Huang HL, Hu YX, Heymsfield AJ, Reichardt J. Geometrical-optics solution to light scattering by droxtal ice crystals. Appl. Opt 2004; 43:2490-2499.

13. Yang $\mathrm{P}$, Liou KN, Wyser K, Mitchell D. Parameterization of the scattering and absorption properties of individual Ice crystals. J Geophys Res 2000; 105(D4): 4699_ 718.

14. Yang P, Wei H, Huang HL, Baum BA, Hu YX, Kattawar GW, Mishchenko MI, Fu Q. Scattering and absorption property database for nonspherical ice particles in the nearthrough far-infrared spectral region. Appl Opt 2005; 44:5512-23.

15. Liu G. A database of microwave single-scattering properties for nonspherical ice particles. Bull Amer Meteor Soc 2008; 89:1563-70.

16. Kim MJ. Single scattering parameters of randomly oriented snow particles at microwave frequencies. J Geophys Res 2006;111, D14201, doi:10.1029/2005JD006892.

17. Hong G, Yang P, Baum BA, Heymsfield AJ, Weng F, Liu Q, Heygster G, Buehler SA. Scattering database in the millimeter and submillimeter wave range of $100-1000 \mathrm{GHz}$ for nonspherical Ice particles. J Geophys Res 2009; 114, D06201, doi: 10.1029/2008JD010451.

18. Baum BA, Yang P, Heymsfield AJ, Platnick S, King MD, Hu YX, Bedka SM, Bulk Scattering Properties for the Remote Sensing of Ice Clouds. II: Narrowband Models. J Appl Meteor 2005; 44:1896-911. 
19. Baum BA, Yang P, Heymsfield AJ, Bansemer A, Merrelli A, Schmitt C, Wang C. Ice cloud bulk single-scattering property models with the full phase matrix at wavelengths from 0.2 to $100 \mu \mathrm{m}$. J Quant Spectrosc Radiat Tansf 2005; 146:123-39.

20. Liu C, Yang P, Minnis P, Loeb N, Kato S, Heymsfield A, Schmitt C. A two-habit model for the microphysical and optical properties of ice clouds.AtmosChemPhys2014; 14:13719-13737, doi:10.5194/acp-14-13719-2014.

21. Yang P, Bi L, Baum BA, Liou KN, Kattawar GW, Mishchenko MI, Cole B. Spectrally consistent scattering, absorption, and polarization properties of atmospheric ice crystals at wavelengths from 0.2 to $100 \mu \mathrm{m}$. J Atmos Sci 2013; 70:330-47.

22. Yang P, Liou KN, Wyser K, Mitchell D. Parameterization of the scattering and absorption properties of individual ice crystals. J Geophys Res, 2000; 105 (D4): 4699718.

23. Yurkin MA, Hoekstra AG. The discrete dipole approximation: An overview and recent developments. J Quant Spectrosc Radiat Transf 2007; 106: 558-89.

24. Nussenzveig HM. Diffraction effects in semi-classical scattering. New York: Cambridge University Press; 1992.

25. Cole BH, Yang P, Baum BA, Riedi J, C-Labonnote L. Ice particle habit and surface roughness derived from PARASOL polarization measurements. Atmos Chem Phys 2013; 14: $3739-50$.

26. Yi B, Yang P, Liou KN, Minnis P, Penner JE. Simulation of the global contrail radiative forcing: A sensitivity analysis. Geophys Res Lett 2012; 39: L00F03, doi: 10.1029/2012GL054042. 
27. Platnick S, Meyer KG, King MD, Wind G, Amarasinghe N, Marchant B, Arnold GT, Zhang Z, Hubanks PA, Holz RE, Yang P, Ridgway WL, Riedi J. The MODIS cloud optical and microphysical products: Collection 6 updates and examples from Terra and Aqua. IEEE Trans Geosci Remote Sens, 55, 502-525, doi:10.1109/TGRS. 2016.2610522 .

28. Holz RE, Platnick S, Meyer K, Vaughan M, Heidinger A, Yang P, Wind G, Dutcher S, Ackerman S, Amarasinghe N, Nagle F, Wang C. Resolving ice cloud optical thickness biases between CALIOP and MODIS using infrared retrievals. Atmos Chem Phys 2016; 16: 5075-90.

29. Arnott WP, Dong YY, Hallett J. Extinction efficiency in the infrared $(2-18 \mu \mathrm{m})$ of laboratory ice clouds: observations of scattering minima in the Christiansen bands of ice. Appl Opt 1995; 34:541-51.

30. Yang P, Liou KN, Arnott WP. Extinction efficiency and single-scattering albedo of ice crystals in laboratory and natural cirrus clouds. J Geophys Res, 1997; 102:21825-35.

31. Johnson BR. Invariant imbedding T-matrix approach to electromagnetic scattering. Appl Opt 1988;27:4861-7.

32. Mishchenko MI. Light scattering by randomly oriented axially symmetric particles. J Opt Soc Am A 1991;8:871-82.

33. Yee SK. Numerical solution of initial boundary value problems involving Maxwell's equations in isotropic media. IEEE Trans Antennas Propag 1966;14:302-7. 
34. Sun W, Fu Q, Chen Z. Finite-difference time-domain solution of light scattering by dielectric particles with perfectly matched layer absorbing boundary conditions. Appl Opt 1999;38:3141-51.

35. Um J, McFarquhar GM. Optimal numerical methods for determining the orientation averages of single-scattering properties of atmospheric ice crystals. J Quant Spectrosc Radiat Transf 2013; 127: 207-223.

36. Bi L, Yang P. High-frequency extinction efficiencies of spheroids: rigorous T-matrix solutions and semi-empirical approximations. Opt Express 2014; 22 (9): 10270-93.

37. Chang PCY, Walker JG, Hopcraft KI. Ray tracing in absorbing media. J Quant Spectrosc Radiat Transf 2005; 70:327-41.

38. Yang P, Liou KN. Effective refractive index for determining ray propagation in an absorbing dielectric particle. J Quant Spectrosc Radiat Transf 2009; 110: 300-6.

39. Yang P, Liou KN. An "exact" geometric-optics approach for computing the optical properties of large absorbing particles. J Quant Spectrosc Radiat Transf 2009; 110: 116277.

40. Van de Hulst HC. The spherical albedo of a planet covered with a homogeneous cloud layer. Astron Astrophys 1974;35:209-214.

41. Ding J, Bi L, Yang P, Kattawar G, Weng F, Liu Q, Greenwald T. Single-scattering properties of ice particles in the microwave regime: temperature effect on the refractive index with implications in remote sensing. J Quant Spectrosc Radiat Transf (in press) 


\section{Appendix A: Treatment of Inhomogeneous Waves}

This section documents the formulas used in a new version of the IGOM code to compute the optical properties. Consider a general inhomogeneous plane wave propagating in a medium with a refractive index $m=m_{r}+i m_{i}$ :

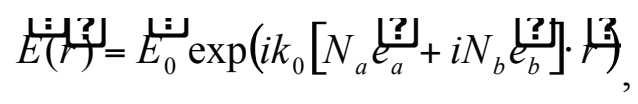

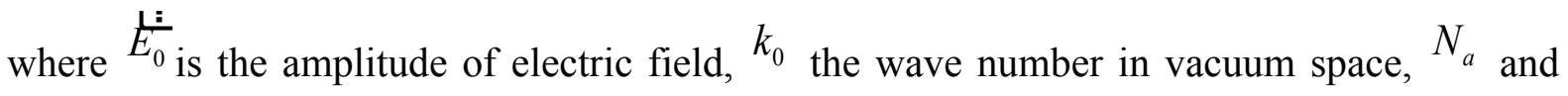
$N_{b}$ the real part and the imaginary part of effective refractive indices, respectively, ${ }_{a}^{\frac{f}{q}}$ the wave propagation direction, and $\frac{\mathfrak{s}}{e_{b}}$ determines the constant-amplitude wave front . Eq. (A1) must satisfy the vector Helmholtz equation in the form

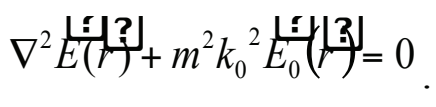

Substituting Eq. (A1) into Eq. (A2), we have

$$
\begin{aligned}
& N_{a}^{2}-N_{b}^{2}=m_{r}^{2}-m_{i}^{2}, \\
& N_{a} N_{b} \underset{a}{\mathscr{4}} \underset{e_{b}}{\underline{4}}=m_{r} \cdot m_{i} .
\end{aligned}
$$

If the medium is non-absorptive, i.e., $m_{i}=0$, there are two sets of solutions from Eq. (A3) and (A4), given by

$$
\begin{gathered}
N_{a}=m_{r}, N_{b}=m_{i}=0 \\
N_{a}^{2}-N_{b}^{2}=m_{i}^{2}, \stackrel{? !}{e_{a}} \stackrel{?}{e_{b}=0} .
\end{gathered}
$$

The wave corresponding to Eq. (A5) is the homogeneous wave. In the case of Eq. (A6), the wave is inhomogeneous in a non-absorptive medium. A straightforward example is the 
evanescent wave arising from total internal reflection. For an absorbing particle $\left(m_{i} \neq 0\right)$, consider a beam (constant phase front) emerging from the particle to air. The amplitude of the electric field within the cross section in air is nonuniform because the path length in the particle is generally variable except in certain special cases such as a parallel slab. Because $e_{a}^{\mathfrak{L}} \cdot \underline{e_{b}}=0$, the amplitude of the electric field in air increases or decreases along a vector

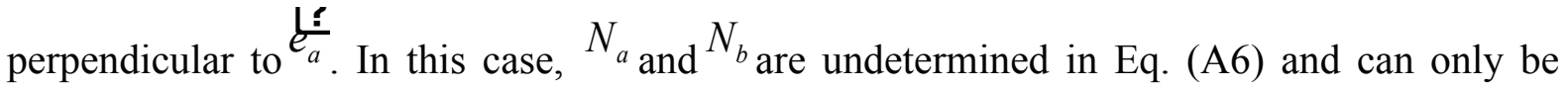
defined through boundary conditions.

For an inhomogeneous wave, a complex wave vector can be defined to be

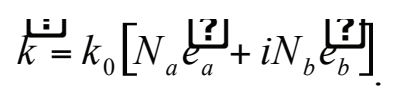

It can either be defined as

$$
\stackrel{!}{k}=m k_{0} \hat{k}
$$

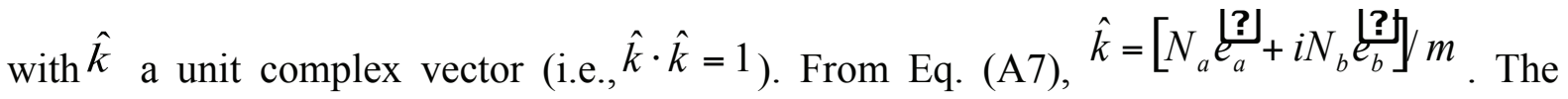
electric-field vector associated with inhomogeneous waves can have a component along the

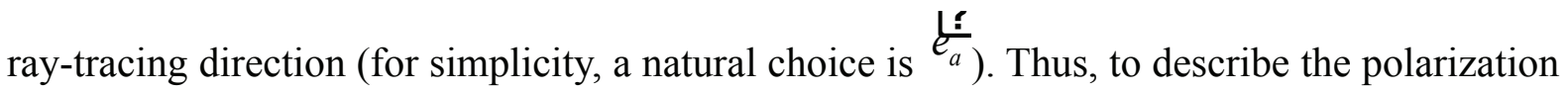
state, three components are required to describe the field oscillating along three orthogonal unit vectors,

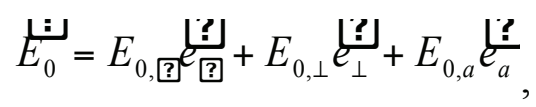




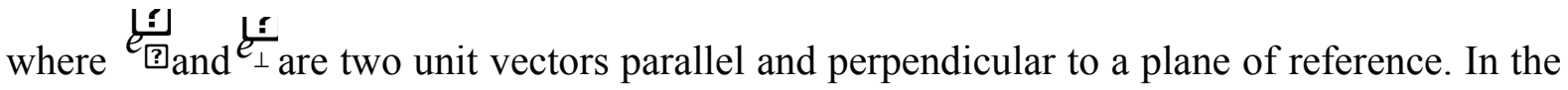
following, we do not use Eq. (A9) for the description of field polarization, but introduce two complex unit vectors to specify the electric field vector:

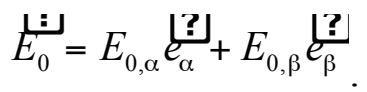

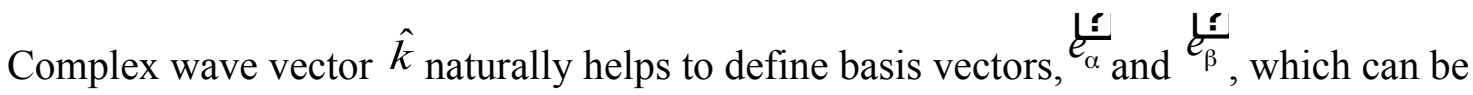
defined by

$$
e_{\alpha}^{?}=\frac{\hat{k} \times \stackrel{? !}{e_{b}}}{\sqrt{\left(\hat{k} \times \frac{?}{e_{b}}\right)\left(\hat{k} \times \frac{?}{e_{b}}\right)}}, \frac{?}{e_{\beta}}=\hat{k} \times \frac{?}{e_{\alpha}}
$$

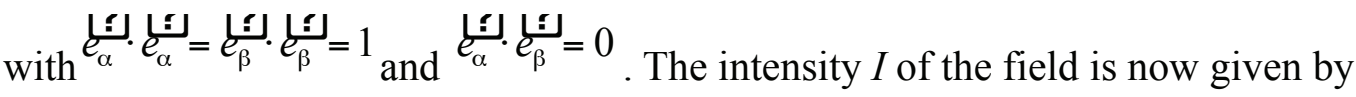

$$
I=0.5\left(\left|E_{0, \alpha}\right|^{2} \vec{e}_{\alpha}^{*} \cdot \vec{e}_{\alpha}+\left|E_{0, \beta}\right|^{2} \vec{e}_{\beta}^{*} \cdot \vec{e}_{\beta}\right) .
$$

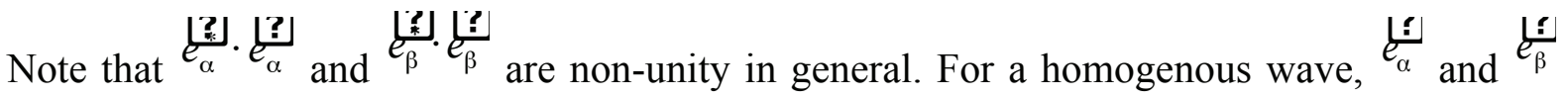
are arbitrary mutual orthogonal real vectors perpendicular to the wave vector.

To obtain Snell's law and Fresnel's formula, the incident wave, reflected wave and refracted/ transmitted wave are assumed to have the following formalism:

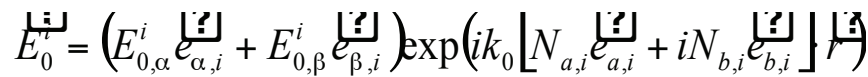

$$
\begin{aligned}
& \vec{E}_{0}^{r}=\left(E_{0, \alpha}^{r} \vec{e}_{\alpha, r}+E_{0, \beta}^{r} \vec{e}_{\beta, r}\right) \exp \left(i k_{0}\left[N_{a, r} \vec{e}_{a, r}+i N_{b, r} \vec{e}_{b, r}\right] \cdot \vec{r}\right)
\end{aligned}
$$

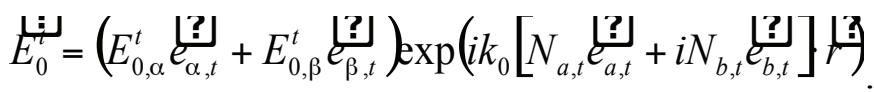


Consider the phase-matching condition,

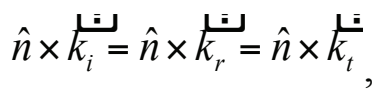

and

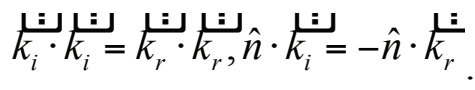

We have the following Snell's law:

$$
\begin{aligned}
& N_{a, i}=N_{a, r}, N_{b, i}=N_{b, r},
\end{aligned}
$$

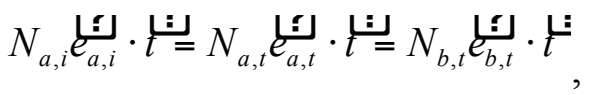

where

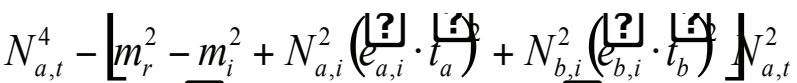

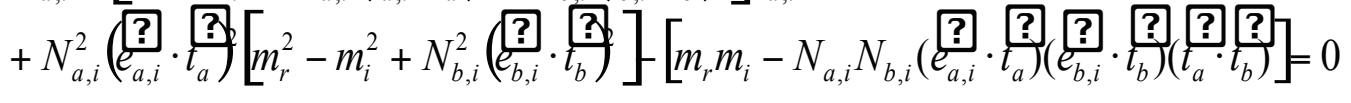

Note that the plane of incidence previously defined in the case of a homogenous wave cannot be easily specified, because $\frac{\underline{T}}{e_{a}}, e_{b}$ and the normal direction of the local flat surface are no longer coplanar. Thus, the traditional TM and TE modes do not exist. Following Chang et al. [38] to define Fresnel's formula, we have the PE mode

$$
r_{\beta}=\frac{K_{i}-K_{t}}{K_{i}+K_{t}}, t_{\beta}=\frac{2 K_{i}}{K_{i}+K_{t}}
$$

and the PM mode 


$$
r_{\alpha}=\frac{m_{t}^{2} K_{i}-m_{i}^{2} K_{t}}{m_{t}^{2} K_{i}+m_{i}^{2} K_{t}}, t_{\alpha}=\frac{2 m_{i} m_{t} K_{i}}{m_{t}^{2} K_{i}+m_{i}^{2} K_{t}},
$$

where $K_{i}=\hat{n} \cdot \hat{k}_{i}, K_{t}=\hat{n} \cdot \hat{k}_{t}$ Eq. (A1)-(A22) provide a self consistent formalism for ray tracing in an absorbing or non-absorbing medium in the new implementation of the IGOM.

\section{Appendix B: A Proof of Asymptotic Extinction}

\section{Efficiency}

In the case of an absorbing particle, the asymptotic amplitude scattering matrix is determined by diffraction and external reflection. According to the optical theorem, the extinction efficiency can be computed from the forward scattering amplitude and should be 2, but this statement is not obvious from the electric field volume integral equations. Here, we provide an analytical proof of the asymptotic extinction efficiency and, particularly, illustrate the importance of treating the nature of inhomogeneous waves in order to obtain the correct value.

Starting from an electric-field volume equation, the amplitude scattering matrix can be obtained as follows

$$
\left[\begin{array}{ll}
S_{2} & S_{3} \\
S_{4} & S_{1}
\end{array}\right]=\sum_{i=1}^{N_{f}} D_{i} \frac{\left(m^{2}-1\right) \cos \theta_{t}}{N_{a}+i \widetilde{N}_{b}-\hat{r} \cdot \hat{e}_{t}}\left[\begin{array}{cc}
\widetilde{S}_{2} & \widetilde{S}_{3} \\
\widetilde{S}_{4} & \widetilde{S}_{1}
\end{array}\right],
$$


where ${ }^{N_{f}}$ is the total number of facets that face the incident wave, ${ }^{\theta_{t}}$ the refraction angle, $\widetilde{N}_{b}=N_{b} \cos \theta_{t}, \hat{r}$ the scattering direction, $\hat{e}_{t}$ the direction of the refracted wave, and $D_{i}$ is the shape factor associated with the i-th local facet. In the direct forward scattering direction,

$$
\left[\begin{array}{ll}
S_{2} & S_{3} \\
S_{4} & S_{1}
\end{array}\right]=\frac{k^{2}}{4 \pi} \sum_{i=1}^{N_{f}} S_{i} \frac{\left(m^{2}-1\right) \cos \theta_{t}}{N_{a}+i \widetilde{N}_{b}-\cos \left(\theta_{i}-\theta_{t}\right)} T_{\beta}\left[\begin{array}{ll}
1 & 0 \\
0 & 1
\end{array}\right]
$$

where $S_{i}$ is the area of the i-th local facet, ${ }_{i}$ the incident angle and $T_{\beta}$ the transmission coefficient, given in the form

$$
T_{\beta}=\frac{2 \cos \theta_{i}}{\cos \theta_{i}+m \cos \tilde{\theta}_{t}}=\frac{2 \cos \theta_{i}}{\cos \theta_{i}+N_{a} \cos \theta_{t}+i N_{b}}
$$

In Eq. (B3), $\tilde{\theta}_{t}$ is the complex refraction angle. Eq. (B2) is simplified as

$$
\left[\begin{array}{ll}
S_{2} & S_{3} \\
S_{4} & S_{1}
\end{array}\right]=\frac{k^{2}}{4 \pi} \sum_{i=1}^{N} S_{i} \frac{2\left(m^{2}-1\right) \cos \theta_{t} \cos \theta_{i}}{\left[N-\cos \left(\theta_{i}-\theta_{t}\right)\right]\left[\cos \theta_{i}+m \cos \tilde{\theta}_{t}\right.}-\left[\begin{array}{ll}
1 & 0 \\
0 & 1
\end{array}\right]
$$

The denominator can be first expanded into multiple terms, represented as follows 


$$
\begin{aligned}
& {\left[N-\cos \left(\boldsymbol{\theta}_{t}-\boldsymbol{\theta}_{i}\right)\right]\left[\cos \boldsymbol{\theta}_{i}+m \cos \tilde{\boldsymbol{\theta}}_{t}\right] } \\
= & {\left[N-\cos \left(\boldsymbol{\theta}_{t}-\boldsymbol{\theta}_{i}\right)\right]\left[\cos \boldsymbol{\theta}_{i}+N \cos \boldsymbol{\theta}_{t}+i \tilde{N}_{b} \tan \boldsymbol{\theta}_{t} \sin \boldsymbol{\theta}_{t}\right] } \\
= & {\left[N-\cos \left(\boldsymbol{\theta}_{t}-\boldsymbol{\theta}_{i}\right)\right]\left[\cos \boldsymbol{\theta}_{i} / \cos \boldsymbol{\theta}_{t}+N+i \tilde{N}_{b} \tan ^{2} \boldsymbol{\theta}_{t}\right] \cos \boldsymbol{\theta}_{t} } \\
= & {\left[N \cos \boldsymbol{\theta}_{i} / \cos \boldsymbol{\theta}_{t}+N^{2}+i N_{a} \tilde{N}_{b} \tan ^{2} \boldsymbol{\theta}_{t}-\tilde{N}_{b}^{2} \tan ^{2} \boldsymbol{\theta}\right.} \\
& \left.-\cos ^{2} \boldsymbol{\theta}_{i}-\tan \boldsymbol{\theta}_{t} \sin \boldsymbol{\theta}_{i} \cos \boldsymbol{\theta}_{i}-N \cos \left(\boldsymbol{\theta}_{t}-\boldsymbol{\theta}_{i}\right)-i \tilde{N}_{b} \tan ^{2} \boldsymbol{\theta}_{t} \cos \left(\boldsymbol{\theta}_{t}-\boldsymbol{\theta}_{i}\right)\right] \cos \boldsymbol{\theta}_{t}
\end{aligned}
$$

Because

$$
\begin{array}{r}
N \cos \left(\theta_{t}-\theta_{i}\right)=N \cos \theta_{t} \cos \theta_{i}+\sin ^{2} \theta_{i}+i N_{a} \tilde{N}_{b} \sin ^{2} \theta_{t} \\
i \widetilde{N}_{b} \tan ^{2} \theta_{t} \cos \left(\theta_{t}-\theta_{i}\right)=i \widetilde{N}_{b} \tan \theta_{t} \sin \theta_{t} \cos \theta_{i}+i N_{a} \widetilde{N}_{b} \tan ^{2} \theta_{t} \sin ^{2} \theta_{t}
\end{array}
$$

we then have

$$
\begin{aligned}
& \left.\left[N-\cos \left(\theta_{t}-\theta_{i}\right)\right] \cos \theta_{i}+m \cos \tilde{\theta}_{t}\right]=\left[N^{2}-\widetilde{N}_{b}^{2} \tan ^{2} \theta_{t}-1\right\rfloor \operatorname{os} \theta_{t} \\
& +\cos \theta_{t}\left[N \cos \theta_{i} / \cos \theta_{t}+i N_{a} \widetilde{N}_{b} \tan ^{2} \theta_{t}-\tan \theta_{t} \sin \theta_{i} \cos \theta_{i}\right. \\
& -N \cos \theta_{t} \cos \theta_{i}+i N_{a} \tilde{N}_{b} \sin ^{2} \theta_{t}-i \widetilde{N}_{b} \tan \theta_{t} \sin \theta_{t} \cos \theta_{i}-i N_{a} \widetilde{N}_{b} \tan ^{2} \theta_{t} \sin ^{2} \theta_{t}- \\
& =\left[N^{2}-\widetilde{N}_{b}^{2} \tan ^{2} \theta_{t}-1\right] \operatorname{os} \theta_{t}+\cos \theta_{t}\left[N \cos \theta_{i} / \cos \theta_{t}-\tan \theta_{t} \sin \theta_{i} \cos \theta_{i}\right. \\
& \left.-N \cos \theta_{t} \cos \theta_{i}-i \widetilde{N}_{b} \tan \theta_{t} \sin \theta_{t} \cos \theta_{i}\right]
\end{aligned}
$$


The second term in the last expression of Eq. (B8) is zero, which can be proved with the following derivation,

$$
\begin{aligned}
& \cos \theta_{t}\left[N \cos \theta_{i} / \cos \theta_{t}-\tan \theta_{t} \sin \theta_{i} \cos \theta_{i}\right. \\
& \left.-N \cos \theta_{t} \cos \theta_{i}-i \widetilde{N}_{b} \tan \theta_{t} \sin \theta_{t} \cos \theta_{i}\right] \\
& =N \cos \theta_{i}-N_{a} \sin ^{2} \theta_{t} \cos \theta_{i}-N \cos ^{2} \theta_{t} \cos \theta_{i}-i \widetilde{N}_{b} \sin ^{2} \theta_{t} \cos \theta_{i} \\
& =N \sin ^{2} \theta_{t} \cos \theta_{i}-N_{a} \sin ^{2} \theta_{t} \cos \theta_{i}-i \widetilde{N}_{b} \sin ^{2} \theta_{t} \cos \theta_{i} \\
& =0
\end{aligned}
$$

Therefore, we obtain

$$
\left.\left[N-\cos \left(\theta_{t}-\theta_{i}\right)\right] \cos \theta_{i}+m \cos \tilde{\theta}_{t}\right\rfloor\left\lfloor N^{2}-\tilde{N}_{b}^{2} \tan ^{2} \theta_{t}-1\right\rfloor \cos \theta_{t}
$$

By using the following identity,

$$
m^{2}=N^{2}-\tilde{N}_{b}^{2} \tan ^{2} \theta_{t}
$$

we have

$$
\left[\begin{array}{ll}
S_{2} & S_{3} \\
S_{4} & S_{1}
\end{array}\right]=\sum_{i=1}^{N} \frac{k^{2}}{2 \pi}\left[\begin{array}{ll}
1 & 0 \\
0 & 1
\end{array}\right]<S_{i}>=\frac{k^{2}}{2 \pi}\left[\begin{array}{ll}
1 & 0 \\
0 & 1
\end{array}\right] \sum_{i=1}^{N}<S_{i}>=\frac{k^{2}<S>}{2 \pi}\left[\begin{array}{ll}
1 & 0 \\
0 & 1
\end{array}\right],
$$

where $<\mathrm{S}>$ is the projected cross section. Eq. (B11) can be obtained from Eqs. (A3) and (A4). According to the optical theorem, the extinction cross section is obtained as follows: 


$$
C_{e x t}=\frac{2 \pi}{k^{2}}\left[S_{1}+S_{2}\right]=2<S>\text {. }
$$

Thus, the extinction efficiency is given by

$$
Q_{e x t}=\frac{C_{e x t}}{<S>}=2
$$

